\title{
Le train
}

\section{J. Vecsey}

Le TGV démarre à l'heure prévue en ce 24 décembre, et peu importe la destination. Tous les voyageurs sont emmitouflés dans d'épais manteaux. Les accessoires des uns, chapeaux, parapluies, gants, moufles, écharpes, bâtons de ski, anoraks, cannes et béquilles, se mêlent aux sacs, paquets, paniers, valises et baise-en-ville des autres.

Huit passagers se font face dans cette partie du wagon, certains se saluent poliment, d'autres ne répondent au salut des premiers que par un vague signe de tête, d'autres enfin ne daignent pas même réagir.

Chacun trouve son siège, creuse son terrier et investit ce qui sera son territoire pendant les trois prochaines heures. Tous préparent revues, quotidiens, livres, tricot, ordinateurs portables, walkman et autres objets plus ou moins intellectuels. L'adage ne dit-il pas «dis-moi ce que tu lis et je te dirai qui tu es»?

Les premiers assis, qui se font face près de la fenêtre, sont un couple d'âge mûr; à mesurer leurs rides et leurs cheveux blancs, ils doivent tout juste être grands-parents, ils n'échangent que quelques mots discrets, fonctionnels, soufflés à voix basse. Tous deux sont vêtus avec recherche et goût, mais sans aucune fantaisie, l'ennui transpire de tous leurs pores, malgré le froid glacial de l'hiver.

A côté du Monsieur a pris place un jeune homme quelque peu maniéré, dont les vêtements confortables et décontractés dégagent une agréable senteur d'eau de toilette à la mode. Il est déjà perdu dans ses pensées avant le départ $\mathrm{du}$ train, la tête dans les étoiles, et les écouteurs vissés sur les oreilles.

A ses côtés est assise une septuagénaire vaillante, strictement vêtue, portant lunettes et petit chapeau mou, qui n'a abandonné son Loden qu'à regret, tant elle craint le froid. Elle s'empare aussitôt d'un tricot, et bientôt le cliquetis de ses aiguilles se mêlera au ronronnement obsédant des roues glissant sur les rails.

En face d'elle a pris place un jeune homme d'allure «normale», mais qui, en l'observant mieux, est extrêmement hésitant et peu sûr de lui, il regarde autour de lui, inquiet d'être dans la bonne direction, dans le bon train, à la bonne heure, à la bonne place; tout est flou dans sa tête, est-on vraiment le 24 décembre? Tous ces gens qui partent comme lui semblent bien l'indiquer, mais s'il ne s'agissait que d'une gigantesque farce collective?

Le voisin de ce jeune homme est un autre monsieur fringuant et sa toute neuve jeune femme, fraîchement épousée. Ils sont seuls au monde, totalement étanches à l'univers extérieur.

Le huitième occupant du compartiment est un monsieur endimanché et un peu poussiéreux pourtant, dont l'apparence ne laisse en rien deviner le métier, pas plus que l'appartenance sociale. Seule une extravagante moustache attire l'attention sur son visage ordinaire. La tristesse et la déception se lisent sur ses traits épais.

Le train s'ébranle, la grisaille du ciel et la tristesse des bâtisses environnantes complètent la monotonie de ce tableau, véritable nature morte, mouvante pourtant. Seuls des arbres de Noël éclairés, disposés au hasard des rues environnantes égaient le paysage citadin.

Une voix féminine, qui se voudrait douce et céleste, égrenée par des hauts parleurs invisibles, informe les voyageurs qu'ils sont les bienvenus au bar, et rassure chacun sur la destination finale du convoi.

Peu à peu, les maisons se font plus rares, la banlieue prend le pas sur les vieux immeubles, alternant avec des gratte-ciels de verre mêlés à des églises anciennes, égarées dans le désordre de cette architecture urbaine.

La campagne prend ensuite le pas sur la ville, et le convoi traverse des bourgades monotones. La neige éparse recouvre à peine des champs labourés aux profonds sillons, rappelant qu'au dehors, il fait froid. Mais dans le cocon du train, le chauffage assure une agréable température.

Le voyage débute sans encombre, et personne ne soupçonne encore qu'il va se passer quelque chose, personne, sauf Vous, lecteur attentif, qui vous dites que s'il ne se passait rien, cette histoire ne mériterait pas d'être écrite!

Le contrôleur pénètre avec ennui dans le compartiment et réclame les contremarques de tous. Sa pêche sera quelconque, puisque chacun produit le sésame de carton.

Le couple de jeunes mariés manque à l'appel, car ils ont préféré s'éclipser pour boire un café en tête-à-tête, de même que le voyageur triste. 
Avec le temps qui passe, peu à peu les langues se délient, les uns commentent tel article du dernier quotidien, les autres telle course de ski au cours de laquelle décidément les Suisses n'ont pas brillé.

Soudain les lumières vacillent, comme autant de bougies, puis, le train s'arrête lentement, et seule la lueur blafarde de décembre éclaire le convoi. Après quelques minutes de silence, les passagers se regardent, interloqués, que se passe-t-il? Ce n'est visiblement pas normal, et chacun d'échafauder intérieurement les hypothèses les plus vraisemblables: Un tronc d'arbre barrant la voie? Un animal égaré sur les rails? Une gigantesque panne d'électricité? Un passage à niveau défectueux? Un attentat terroriste? Un suicidant désespéré?

Dehors, on ne voit rien, et pas moyen de se pencher par les fenêtres irrémédiablement closes pour deviner l'origine de cette halte intempestive.

Peu à peu, les regards interloqués font place à des commentaires divers et inquiets. L'arrêt inhabituel pousse ces gens à se parler, à avancer leurs avis sur l'origine de cette interruption involontaire.

Le couple de jeunes mariés est revenu, la dame au tricot leur sourit gentiment, et se hasarde à leur dire: Je suis convaincue que ce n'est pas le voyage de noces auquel vous vous attendiez, n'est-ce pas? (elle avait repéré sur leurs bagages un autocollant indiquant «just married» lorsqu'ils sont montés). Bah, répond le frais mari, quelle importance, la vie est devant nous. Sa femme acquiesce. Puisque le débat est lancé, la paire de grands parents s'interroge à haute voix sur l'heure à laquelle ils arriveront à destination, après tout, ils sont attendus, et même s'ils pourront prévenir de leur retard suffisamment tôt, ils se montrent très contrariés.

Le jeune homme aux écouteurs les a retirés pour participer à la conversation, mais lui se montre fataliste. Contre toute attente, il propose même à la femme d'âge mûr lui faisant face de partager sa musique avec lui, en lui tendant une de ses oreillettes: le temps passera ainsi plus vite. Elle accepte par curiosité, et découvre une musique indigeste et résolument contemporaine. C'est du Goebbels, non, non, pas Joseph, rendu tristement célèbre lors de la dernière guerre, mais Heiner, l'auteur contemporain de la musique du prochain ballet que le corps de danse du jeune homme interprétera à l'opéra le mois prochain. Il écoute ces notes sans relâche, pour mieux s'en imprégner, répétant la chorégraphie intérieurement. Après ces quelques mots d'explication, non seulement la musique semble plus facile aux oreilles de la dame, mais le jeune homme maniéré gagne sa sympathie.

La dame n'a pas l'intention de dévoiler à tous que personne ne l'attend à l'arrivée, qu'elle part seule précisément pour fuir sa solitude; elle n'ignore pourtant pas que plus une ville est grande, plus on s'y sent seul, que la fuite en avant n'est pas le moyen d'ouvrir les portes, mais c'est plus fort qu'elle, une force inconnue l'incite à partir, à aller rechercher le temps pourtant inexorablement perdu. Elle ne dira rien de plus.

L'arrêt se prolonge décidément, la journée avance, et à 16 heures, la lumière devient rare; bientôt pardessus et manteaux refont leur apparition avec la chute inattendue du thermomètre: oui, oui, c'est une évidence, le chauffage ne fonctionne plus. La vieille dame tire un paquet de biscuits de son sac, et en offre à tous. Pour tromper la faim autant que pour s'occuper, tous en acceptent, par désœuvrement plus que par gourmandise. Tiens, tiens, elle pourrait bien se prénommer Madeleine, la vieille dame... Alors c'est elle qui va mener le bal désormais.

Le contrôleur passe dans chaque wagon et, tout penaud, annonce d'une voix lasse qu'une gigantesque panne d'électricité est responsable de cet arrêt intempestif. D'où vient-elle? L'histoire ne le dit pas, et après tout, peu importe, même si le monsieur endimanché au regard triste dit avoir lu dans le journal que les intempéries de ce début d'hiver avaient mis les installations électriques de la région à rude épreuve. Les hypothèses peuvent aller bon train, personne ne possède la réponse.

Madeleine ouvre la discussion donc, et tout en tricotant, raconte à qui veut l'entendre, de même qu'à ceux qui ne le souhaitent pas, qu'elle est une institutrice à la retraite (qui l'eut cru?), et qu'elle va rejoindre son fils à l'occasion des fêtes de Noël. Elle vient tout juste d'enterrer son défunt mari, et la liberté retrouvée lui redonne un semblant de jeunesse et d'enthousiasme! (Ca, elle ne le dit pas vraiment!)

Sa carrière a été longue et enrichissante, elle en a croisé de futurs épiciers, patrons, clochards, paysans, pharmaciens, comptables, artistes et autres politiciens. Tous avaient au départ les mêmes chances théoriques, puis la vie, les opportunités, les diverses fortunes, la volonté et le hasard avaient fait le reste. Il lui en était resté cette solide autorité de meneuse dont elle ne pouvait se départir aujourd'hui.

Alors pour respecter la règle, chacun, pour autant qu'il soit d'accord de jouer, va raconter un épisode de sa vie.

Après l'institutrice, c'est le tour des jeunes mariés de parler, eux qui commencent certes une 
vie commune, mais qui ont chacun un passé; lui est informaticien, et outre les jeux de tactique à l'écran et la pratique assidue du sport en canapé, c'est un dévoreur de BD. Mais pour le moment, c'est sa jeune épouse qu'il dévore des yeux. Nous n'en saurons pas plus sur lui, considérons que c'est déjà généreux de sa part d'avoir autant levé le voile sur son propre sujet.

Les passions avouées de sa jeune femme sont: son mari, les enfants. Qu'affectionne-t-elle à part cela? Nous saurons qu'elle est éducatrice, mais rien de ses intérêts ni de son jardin secret...

C'est alors au couple élégant de parler d'eux: leur aspect est terriblement conventionnel, certes, mais quand ils ouvrent la bouche, parlant haut et fort maintenant, leurs compagnons d'infortune vont être surpris: ils sortent de la discrétion pour avouer qu'elle est une diplomate en fonction dans une capitale européenne, et qu'il est son chauffeur, ce qui n'est à priori pas surprenant. Ce qui l'est un peu plus, c'est d'apprendre qu'ils sont mari et femme. Mais ils viennent d'un pays nordique, où la culture est différente, à quelques degrés de latitude d'ici seulement, et ce genre d'union est banal, là-bas. Elle commence alors à raconter qu'en fonction en Russie, il y a deux ans, l'arrêt brutal d'un train avait été provoqué par un attentat terroriste, et si l'intervention militaire des autorités avait certes permis de ne pas céder aux revendications révolutionnaires des assaillants, l'histoire s'était pourtant mal terminée, car des coups de feu, qui se voulaient dissuasifs seulement, avaient été tirés, frappant au hasard et malencontreusement d'innocentes victimes. Elle s'empresse d'ajouter qu'ici, il ne se passe de tel...

La vieille dame encourage alors le jeune homme aux regards inquiets à parler: il s'éclaircit la voix pour raconter son histoire, d'un ton mal assuré: il y a quelques mois, par un bel après-midi dominical, quand la lumière du soleil couchant souligne les contours d'un trait d'ombre, le public était venu nombreux au manège admirer les étalons menés par d'habiles cavaliers.

Son tour venu, il sautait les obstacles avec aisance, tout était facile, rien ne résistait à sa monture. Il s'approchait de la barrière du mur au trot, puis son cheval s'est mis au galop sous l'impulsion de son cavalier pour franchir cet obstacle habituellement banal. Mais cette fois-ci, il était de plus en plus haut, de plus en plus menaçant et... infranchissable. Alors ce fut le fracas des briques factices volant en éclat, le mur brisé en même temps que tous les espoirs de l'homme en devenir.
Sa tête heurte lourdement le sol, tout y explose, malgré la bombe, protection dérisoire... Ses membres ne lui appartiennent plus, il n'est plus qu'un pantin désarticulé, à la merci des lois de la pesanteur.

La suite n'a été qu'une succession de douleur, de travail, de deuils, comme le cheval qu'il ne pourrait plus jamais monter comme avant, les études, qui allaient être bien difficiles à reprendre, les voyages lointains... Comment s'y retrouver devant des tableaux, des panneaux, des pages, tous couverts de hiéroglyphes devenus incompréhensibles, d'étiquettes illisibles? C'était impossible.

L'avenir serait, mais bien différent de celui auquel il était préparé. Alors, après l'hôpital, pour fêter sa liberté toute fraîche et relative, et pour vaincre ses démons, il faisait son premier voyage, seul déjà. Sa famille l'attendait au port, mais avant d'y arriver, que de bruits, que d'éclairs, que de bousculades, quel brouhaha!

Les autres se taisent, mal à l'aise après les confidences du garçon.

Mais la vie continue, et c'est maintenant au monsieur engoncé dans son habit mal coupé et inconfortable de raconter le but de son voyage: il est allé présenter, sans succès, sa dernière invention à un industriel: ce doux dingue, ingénieur de son état, a mis au point une lampe d'un genre nouveau. L'énergie nécessaire au fonctionnement de l'ampoule est fournie par le travail des muscles respiratoires de celui qui l'actionne.

Sa géniale invention n'a pas intéressé l'industriel, car de nos jours, à l'heure du nucléaire, est-ce qu'Eole, ou les poumons, les pauvres cousins du vent, sont vraiment assez puissants pour venir au secours de l'industrie?

Toujours est-il que la nuit est maintenant tombée sur les voyageurs et leur compartiment.

Devant l'insistance de ses compagnons, notre inventeur extirpe de sa valise le volumineux engin. Il le met en place devant lui, se saisit de l'embout, souffle... et une pauvre lumière jaillit de l'ampoule. La flamme vacille au rythme de sa respiration, plus lumineuse à l'aller qu'au retour, mais il ne peut bien sûr plus dire un mot pour insister auprès de ses voisins sur l'importance de se trouvaille!

Qu'à cela ne tienne, ils sont tous bien heureux d'être ainsi à l'abri d'une obscurité angoissante, et ne manquent pas de l'encourager à la moindre faiblesse.

Mais la lumière des plafonniers se rallume, le train repart, et finalement une voix, masculine cette fois, s'excuse platement pour les désagré- 
ments occasionnés par ce retard involontaire et indépendant de la volonté... de qui que ce soit.

Finalement le convoi, tous feux allumés, entre en gare, ceux qui sont attendus retrouvent leurs proches (ah, si les téléphones portables n'existaient pas il faudrait les inventer!), et tous sont heureux d'être arrivés à bon port presque sans complication.

Le voyage se termine sans encombre certes, mais pas sans histoires! L'inventeur du dimanche donne ses coordonnées à la diplomate, qui s'est montrée très intéressée par son appareil, bien utile dans son pays aux mille et une nuits...

La vieille dame est attendue par son fils, accompagné d'un adolescent, traîné à la gare un peu contre son gré pour accueillir sa grand-mère. Après les retrouvailles familiales, le trio se dirige vers l'auto, puis l'institutrice se met à leur expliquer les raisons de ce retard. Elle raconte l'aventure à peu près comme elle s'est passée. Pour la jeune génération, le récit pourrait être relooké comme ceci, sous réserve:

Huit voyageurs se font face dans le tortillard, et tous trouvent le temps un peu long. Ils se font un peu chier, t'sais, comme s'ils avaient voulu être ailleurs. $Y$ en a plusieurs qui sont assez vioques, de l'âge des parents, et même plus.

D'abord, y en a deux qui sont assis près de la fenêtre, avec des fringues vraiment ringard, un couple ancien.

Il y a un jeune, qui a l'air assez cool, il écoute de la zique par ses écouteurs, et à voir sa boule, le sound doit être trop de la balle, t'sais. Plus tard, il la fera même écouter à la nénette qu'est en face de lui.

$Y^{\prime}$ a aussi un autre gars, mais s'il est jeune aussi, lui n'a pas du tout l'air clean, il a vraiment l'air paumé de chez paumé.

Ensuite y'a un couple, elle est vraiment trop cheum, mais bon, l'amour rend aveugle, et on comprend vite qu'ils sont jeunes mariés, vu qu'ils disparaissent rapidement pour s'isoler, t'sais... Bref, ils sont grave in love.

Le huitième mec est un vieux indescriptible, avec une moustache trop cheum, elle aussi. Il est mal fagoté, trop loose, t'sais, pas du tout fashion.

Le train roule, puis tout à coup s'arrête dans un paysage à chier.

Tous sont complètement saoulés par ce voyage de merde, ils voudraient qu'on aille plus vite, go go go! Alentour, le paysage, dont de toute façon tout le monde se fout, est vraiment trop laid. Mais il n'y a rien d'autre à voir, c'est à gerber, et comme les fenêtres ne peuvent pas s'ouvrir, on ne peut même pas poser sa plaque dehors.

L'arrêt est dû à une monstre panne de courant, il fait bientôt nuit, peut-être que la facture d'électricité était trop reuch, alors personne ne l'a payée, t'sais. Pour que personne n'ait trop peur de la nuit, et aussi pour se réchauffer, le monsieur original souffle dans une drôle de machine qui produit de la lumière, bon, un peu tremblante, mais lumière quand même.

C'était trop style.

Et puis le train est reparti, a roulé, est arrivé, et nous voici. 